\title{
Randomized Multi-Channel Interrogation Algorithm for Large-Scale RFID Systems
}

\author{
Amir-Hamed Mohsenian-Rad, Vahid Shah-Mansouri, Vincent W.S. Wong, and Robert Schober \\ Department of Electrical and Computer Engineering, \\ The University of British Columbia, Vancouver, Canada \\ e-mail: $\{$ hamed, vahids, vincentw, rschober $\} @$ ece.ubc.ca
}

\begin{abstract}
A radio frequency identification (RFID) system consists of a set of readers and several objects, equipped with small computer chips, called tags. In a dense RFID system, where several readers are placed together to improve the read rate and correctness, readers and tags can frequently experience packet collision. A common approach to avoid collision is to use a distinct frequency channel for interrogation for each reader. Various multi-channel anti-collision protocols have been proposed for RFID readers. However, due to their heuristic nature, most algorithms may not fully utilize the achievable system performance. In this paper, we develop an optimization-based distributed randomized multi-channel interrogation algorithm, called FDFA, for large-scale RFID systems. For this purpose, we develop elaborate models for reader-to-tag and reader-toreader collision problems. FDFA algorithm is guaranteed to find a local optimum of a max-min fair resource allocation problem to balance the processing load among readers. Simulation results show that FDFA has a significantly better performance than the existing heuristic algorithms in terms of the number of successful interrogations. It also better utilizes the frequency spectrum.
\end{abstract}

\section{INTRODUCTION}

Radio frequency identification (RFID) is an emerging wireless technology which allows objects to be identified automatically. An RFID tag is a small and inexpensive electronic device designed for wireless data transmission. Each tag has a unique ID. It transmits data over the air in response to interrogation signals by an RFID reader. Multiple readers can connect to a back-end system to transfer data for processing or storage [1].

In an RFID system with one reader and several tags, since the reader and the tags share the same wireless channel, tagto-tag collision can occur when multiple tags transmit signals simultaneously to the same reader. This prevents the reader from recognizing any tag. Various tag anti-collision protocols are proposed in [2], [3]. An Aloha-based tag anti-collision scheme has also been standardized by EPCglobal in [4] where the reader begins each interrogation round by informing all the tags about the frame size. Each tag then chooses a time slot at random and transmits only within that time slot.

In several RFID applications (e.g., for inventory checking in a large-scale warehouse), it is necessary to deploy several readers to achieve complete interrogation coverage. In this case, apart from tag collision, reader-to-tag and reader-to-reader collisions may also occur. Reader-to-tag collision occurs when a tag receives signals of comparable strengths from more than one reader simultaneously. This can cause the tag to respond arbitrarily to the readers, leading to incorrect interrogation. Reader-to-reader collision occurs when a reader, which is in the midst of listening to a tag's reply, receives stronger signals from one or more neighboring readers operating at the same frequency simultaneously. This interference can prevent the reader's receiver from decoding the tag's reply successfully.

In a stationary RFID system with synchronized readers and a centralized controller, reader-to-tag and reader-to-reader collisions can be prevented by using a combination of frequency and time division multiple access (FDMA and TDMA) [5]. However, in many practical large-scale RFID systems, centralized coordination and synchronization are difficult to achieve. Therefore, it is of interest to design distributed randomized interrogation schemes such that each reader randomly selects the start time of its interrogation rounds and its operating channel to reduce the probabilities of reader-to-tag and readerto-reader collisions. Related algorithms include naive, random, and carrier sensing protocols [6], the distributed interference avoidance (DIA) algorithm with detect and abort [7], and the slotted listen-before-talk (S-LBT) scheme [8]. However, given the heuristic nature of all of these algorithms, some may not be able to fully utilize the potential capacity of RFID systems. This motivates us to study the multi-channel random access problem in RFID systems within an optimization-based theoretical framework. Our contributions are as follows:

- We formulate an optimization-based multi-channel randomized interrogation problem for large-scale RFID systems. Our objective is to achieve max-min fairness among readers by taking into account reader-to-reader and reader-to-tag interference. Max-min fairness balances the performance and processing load among readers.

- We propose a distributed algorithm to solve the max-min fair optimization problem. Our algorithm, referred to as FDFA, works based on the iterative coordinate ascent mechanism and is guaranteed to reach a local optimal solution of the optimization problem.

- Simulation results show that the FDFA algorithm has a significantly better performance compared to the previously proposed heuristic reader anti-collision algorithms in terms of the number of correct interrogations and fairness among readers. It better utilizes the frequency spectrum. It also converges asynchronously and fast. The max-min fairness objectives at the obtained local optima are very close to the globally optimal values.

The rest of this paper is organized as follows. The system model is described in Section II. Our proposed FDFA algorithm is presented in Section III. Simulation results are presented in Section IV. Conclusions are given in Section V. 


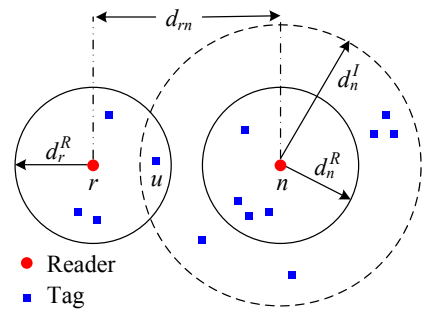

(a) Type 1

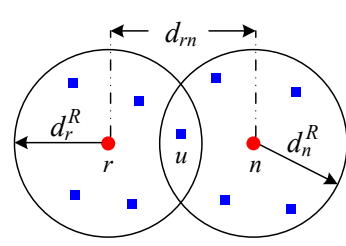

(b) Type 2
Fig. 1. Reader-to-tag collision in an RFID system.

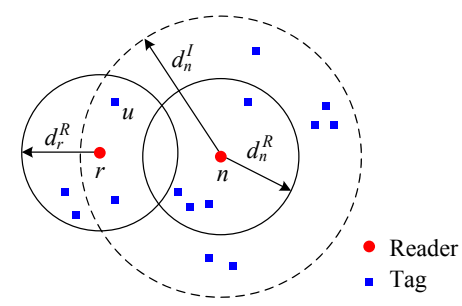

Fig. 2. Reader-to-reader collision in an RFID system.

\section{System ModeL}

\section{A. Reader Collision Problem}

Let $\mathcal{R}$, with size $R=|\mathcal{R}|$, denote the set of all readers. For each $r \in \mathcal{R}$, let $d_{r}^{R}$ denote the read range (or interrogation range) of reader $r$. Reader $r$ can collect information only from those tags which are located within its read range. Let $d_{r}^{I}$ denote the interference range of reader $r$. Reader $r$ 's transmission can interfere the interrogation process of other readers on any tag within its interference range. Two types of reader-to-tag collisions can be distinguished. The first type is shown in Fig. 1(a), where tag $u$ is within the read range of reader $r$ and the interference range of reader $n$ (but not within the read range of reader $n$ ). If both readers use the same channel and transmit simultaneously, tag $u$ cannot correctly decode the message from its reader, i.e., reader $r$. Let $\mathcal{I}_{r}$ denote the set of readers with their interference area (but not their read area) overlapping the read area of reader $r$. We have

$$
\mathcal{I}_{r}=\left\{n: d_{r}^{R}+d_{n}^{R}<d_{r n}<d_{r}^{R}+d_{n}^{I}, n \in \mathcal{R}\right\},
$$

where $d_{r n}$ denotes the Euclidean distance between $r$ and $n$. The first type of reader-to-tag collision can be avoided if readers $r$ and $n$ operate at different frequencies or times [5].

The second type of reader-to-tag collision is shown in Fig. 1(b), where tag $u$ is within the read range of both readers $r$ and $n$. In RFID systems, tags have low functionality and do not have frequency selectivity [4]. Even if readers $r$ and $n$ operate at different channels, tag $u$ cannot decode the interrogation message correctly when both readers transmit simultaneously. Let $\mathcal{S}_{r}$ denote the set of readers which have shared (i.e., overlapped) read area with reader $r$. We have

$$
\mathcal{S}_{r}=\left\{n: d_{r n}<d_{r}^{R}+d_{n}^{R}, n \in \mathcal{R}\right\} .
$$

The second type of reader-to-tag collision can be avoided by having neighboring readers operate at different time slots. Notice that having neighboring readers operate at different frequencies cannot avoid this type of collision. Also notice

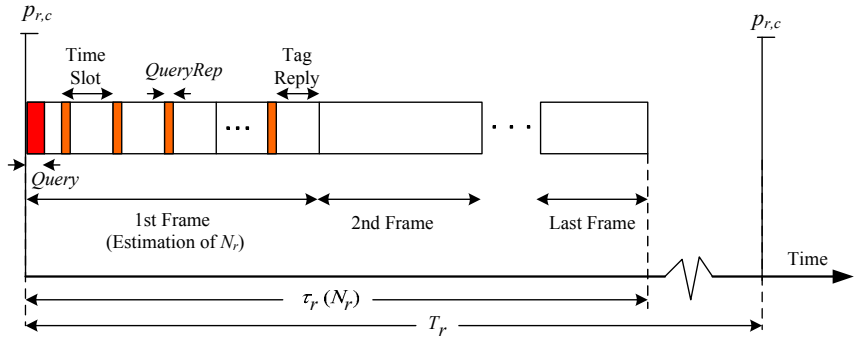

Fig. 3. An interrogation interval, with length $T_{r}$ time units, for reader $r$.

that since $d_{n}^{I} \geq d_{n}^{R}$ for all $n \in \mathcal{R}$, we have

$$
\mathcal{I}_{r} \cap \mathcal{S}_{r}=\{\}, \quad \forall r \in \mathcal{R} .
$$

Furthermore, reader-to-reader collision occurs when reader $n$ transmits while reader $r$ is receiving a message from tag $u$ (see Fig. 2). Let $\mathcal{V}_{r}$ denote the set of readers which have reader $r$ within their interference range. That is,

$$
\mathcal{V}_{r}=\left\{n: d_{r n}<d_{n}^{I}, n \in \mathcal{R}\right\}
$$

Reader-to-reader collision can be avoided if readers operate at different frequencies or time slots [5].

\section{B. RFID Multi-Channel Medium Access Control}

Let $\mathcal{C}$, with size $C=|\mathcal{C}|$, denote the set of available orthogonal frequency channels. For multi-channel random access, we assume that the RFID medium access control (MAC) layer complies with the EPCglobal Class-1 Generation-2 (C1G2) standard [9]. Each reader $r \in \mathcal{R}$ attempts to perform the interrogation process every $T_{r}$ time units. At the beginning of each interrogation interval (see Fig. 3), reader $r$ randomly chooses to start an interrogation process over frequency channel $c \in \mathcal{C}$ with interrogation probability $p_{r, c} \in[0,1]$. We have

$$
\sum_{c \in \mathcal{C}} p_{r, c} \leq 1, \quad \forall r \in \mathcal{R} .
$$

In our model, the proposed randomized multi-channel interrogation algorithm (see Section III) controls reader-to-reader and reader-to-tag collisions, while $\mathrm{C} 1 \mathrm{G} 2 \mathrm{MAC}$ is used to avoid tag-to-tag collision. According to C1G2 MAC [9], if reader $r$ decides to start an interrogation round, it initiates its $1 \mathrm{st}$ interrogation frame by broadcasting a Query message, which includes the number of time slots within the frame. The rest of the frame is then divided into several small time slots, each starting with a QueryRep message to coordinate the timing of sending the reply messages by the tags. Each tag randomly chooses to send its reply back to reader $r$ at one of the available time slots. By the end of the 1st frame, reader $r$ receives the replies from a subset of the existing tags. Based on that, it estimates the number of tags inside its read area, denoted by $N_{r}$, e.g., using the technique in [2]. Given the estimate of $N_{r}$, reader $r$ continues initiating more interrogation frames (i.e., 2nd frame, etc) until it can assure, with a certain statistical confidence, that it has obtained the information from all the tags inside its read area. We denote the duration of each interrogation process by $\tau_{r}\left(N_{r}\right)$ as shown in Fig. 3 .

In practice, we can assume that the interrogation interval is the same for all readers: $T_{r}=T$ for all $r \in \mathcal{R}$ where $T>0$. However, the interrogation intervals of different readers 


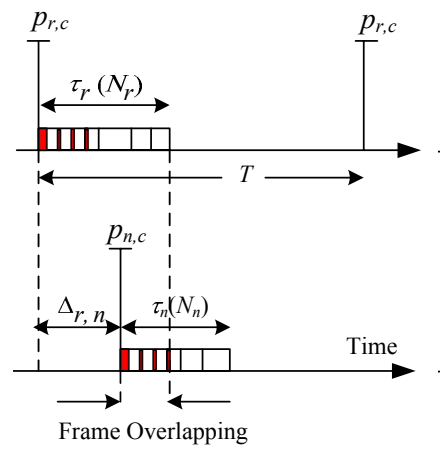

(a) $\Delta_{r, n}<\tau_{r}\left(N_{r}\right)$

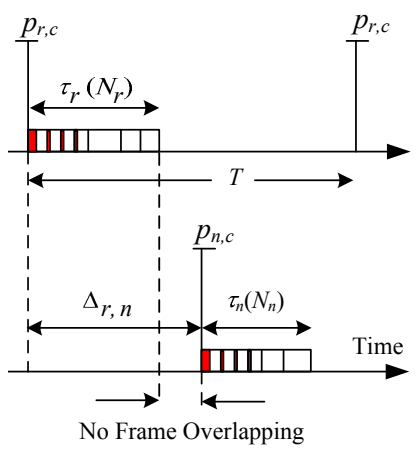

(b) $\Delta_{r, n}>\tau_{r}\left(N_{r}\right)$
Fig. 4. Asynchronism between interrogation intervals for readers $r$ and $n$. In scenario (a), there is a time overlapping between the interrogation processes of readers $r$ and $n$. In this case, asynchronism factor $\Delta_{r, n}<\tau_{r}\left(N_{r}\right)$. In scenario (b), there is no time overlapping between the interrogation processes of readers $r$ and $n$. In this case, asynchronism factor $\Delta_{r, n}>\tau_{r}\left(N_{r}\right)$.

may not be synchronized. Therefore, the interrogation process for different readers may not start at the same time. We can assume that for each pair of readers $r, n \in \mathcal{R}$, there exists a time difference $\Delta_{r, n}$, called the asynchronism factor, between the interrogation intervals of readers $r$ and $n$. The asynchronism factor $\Delta_{r, n}$ is shown in Fig. 4. Clearly,

$$
-T<\Delta_{r, n}<T, \quad \forall r, n \in \mathcal{R} .
$$

Note that $\Delta_{r, n}=-\Delta_{n, r}$. In general, depending on the RFID application, reader $r$ may not always be able to estimate $\Delta_{r, n}$. In this paper, unless stated otherwise, we consider the general case, where the asynchronism factors are not known.

In Fig. 4, reader $r$ randomly decides to start an interrogation on one of the channels every $T$ time units. If the interrogation intervals of readers $r$ and $n$ have time overlapping, as in Fig. 4(a), then a reader-to-tag collision (type 1 or type 2) occurs for $n \in \mathcal{I}_{r}$ or $n \in \mathcal{S}_{r}$, respectively. Reader-to-reader collision can occur if $n \in \mathcal{V}_{r}$. If interrogation intervals have no overlapping, as in Fig. 4(b), reader collisions will not occur.

Let $\boldsymbol{p}=\left(p_{r, c}, \forall r \in \mathcal{R}, c \in \mathcal{C}\right)$ denote the interrogation probability vector. Let $P_{r}^{\text {succ }}(\boldsymbol{p})$ denote the probability of completing a successful interrogation interval by reader $r \in$ $\mathcal{R}$. That is, the probability that reader $r$ starts an interrogation interval without experiencing either reader-to-reader or readerto-tag collisions. We can show the following key result.

Theorem 1: Assume that the interrogation interval $T$ is selected large enough to be at least twice as large as the length of any interrogation process among readers. That is, let

$$
\max _{r \in \mathcal{R}} \tau_{r}\left(N_{r}\right) \leq T / 2 \text {. }
$$

In that case, for each reader $r \in \mathcal{R}$, we have

$$
\begin{aligned}
P_{r}^{\text {succ }}(\boldsymbol{p})= & \left(\prod_{n \in \mathcal{S}_{r}}\left(1-\gamma_{r, n} \sum_{e \in \mathcal{C}} p_{n, e}\right)\right) \\
& \times\left(\sum_{c \in \mathcal{C}} p_{r, c}\left(\prod_{m \in \mathcal{I}_{r}}\left(1-\gamma_{r, m} p_{m, c}\right)\right)\right),
\end{aligned}
$$

where $\gamma_{r, n}$ denotes the probability that the interrogation processes of readers $r$ and $n$ have any time overlapping:

$$
\gamma_{r, n}=\frac{\tau_{r}\left(N_{r}\right)+\tau_{n}\left(N_{n}\right)}{T}
$$

If $\Delta_{r, n}$ is known (by clock synchronization of readers), then $\gamma_{r, n}=1$ if $-\tau_{n}\left(N_{n}\right)<\Delta_{r, n}<\tau_{r}\left(N_{r}\right)$; and $\gamma_{r, n}=0$ otherwise.
The proof of Theorem 1 is given in Appendix A. Since $\mathcal{V}_{r} \subseteq \mathcal{I}_{r} \cup \mathcal{S}_{r}$ for each $r \in \mathcal{R}$, if neither type 1 nor type 2 reader-to-tag collisions occur, then reader-to-reader collision will not happen either. Thus, the set $\mathcal{V}_{r}$ does not appear in (8). It is easy to verify that if $\tau_{r}\left(N_{r}\right)>T / 2$ for all $r \in \mathcal{R}$, then we can simply set $\gamma_{r, n}=1$ for each $r \in \mathcal{R}$ and any $n \in \mathcal{I}_{r}$.

There are several ways to determine the duration of an interrogation process. In the EPCglobal C1G2 system, we have $\tau_{r}\left(N_{r}\right)=e N_{r} \tau^{\text {slot }} \approx 2.72 N_{r} \tau^{\text {slot }}$ for $r \in \mathcal{R}$. Here, $\tau^{\text {slot }}$ denotes the duration of each time slot in a $\mathrm{C} 1 \mathrm{G} 2$ frame. On average, we have $\tau^{\text {slot }}=850 \mu \mathrm{s}$ [4]. Using enhanced dynamic framed slotted Aloha [10] with known $N_{r}$, we have $\tau_{r}\left(N_{r}\right)=3 N_{r} \tau^{\text {slot }}$.

\section{Problem Formulation}

Let $\mathcal{P}$ denote the feasible set of interrogation probabilities:

$$
\mathcal{P}=\left\{\boldsymbol{p}: \sum_{c \in \mathcal{C}} p_{r, c} \leq 1, p_{r, c} \in[0,1], \forall r \in \mathcal{R}, c \in \mathcal{C}\right\} .
$$

In this paper, our goal is to select $\boldsymbol{p} \in \mathcal{P}$ to increase the probability of successful interrogation for all readers to achieve max-min fairness among readers. As a result, the processing load is evenly distributed among all the readers. A vector of feasible interrogation probabilities $\boldsymbol{p} \in \mathcal{P}$ is defined to be max-min fair if any success probability $P_{r}^{\text {succ }}$ cannot increase without decreasing some $P_{n}^{\text {succ }}$ which is smaller than or equal to $P_{r}^{\text {succ }}$. To obtain max-min fairness, it suffices to solve the following non-linear optimization problem [11, Lemma 3]:

$$
\underset{\boldsymbol{p} \in \mathcal{P}}{\operatorname{maximize}} \sum_{r \in \mathcal{R}} f_{\alpha}\left(P_{r}^{\text {succ }}(\boldsymbol{p})\right)
$$

where

$$
f_{\alpha}\left(P_{r}^{\text {succ }}\right)=-\alpha^{-1}\left(P_{r}^{\text {succ }}\right)^{-\alpha}
$$

and $\alpha>0$ is large (e.g., $\alpha \geq 10$ ). Function $f_{\alpha}$ is called a utility function. Next, we propose an algorithm to solve problem (P1).

\section{FDFA ALGORITHM}

Although the utilities in (11) are concave functions in $P_{r}^{\text {succ }}$ for $\alpha>0$, problem (P1) is not a convex optimization problem with respect to $\boldsymbol{p}$, due to the product forms in (8). Thus, finding the optimal solutions of problem (P1) is not easy in general.

For each reader $r \in \mathcal{R}$, we define $\boldsymbol{p}_{r}=\left(p_{r, c} \forall c \in \mathcal{C}\right)$. Here, the key idea is to use the iterative coordinate ascent method [12] to locally update the interrogation probabilities for each reader. In this method, we fix all of the components of vector $\boldsymbol{p}$ to some values, except for those components corresponding to one randomly selected reader (e.g., $\boldsymbol{p}_{r}$ for reader $r$ ). Then, in a local optimization procedure, we maximize the objective function of problem (P1) with respect to $\boldsymbol{p}_{r}$. This procedure is repeated, leading to an iterative algorithm.

\section{A. Local Problem}

Let $\boldsymbol{p}_{-r}=\left(p_{n, c}, \forall n \in \mathcal{R} \backslash\{r\}, c \in \mathcal{C}\right)$ denote the vector of interrogation probabilities of all readers other than reader $r$. Consider the following local problem for reader $r \in \mathcal{R}$ :

$$
\begin{aligned}
\underset{\boldsymbol{p}_{r} \succeq \mathbf{0}}{\operatorname{maximize}} & f_{\alpha}\left(P_{r}^{\text {succ }}\left(\boldsymbol{p}_{r}, \boldsymbol{p}_{-r}\right)\right) \\
& +\sum_{n: r \in \mathcal{S}_{n}} f_{\alpha}\left(P_{n}^{\text {succ }}\left(\boldsymbol{p}_{r}, \boldsymbol{p}_{-r}\right)\right) \\
& +\sum_{m: r \in \mathcal{I}_{m}} f_{\alpha}\left(P_{m}^{\text {succ }}\left(\boldsymbol{p}_{r}, \boldsymbol{p}_{-r}\right)\right) \\
& +\sum_{k: r \notin \mathcal{S}_{k} \cup \mathcal{I}_{k}} f_{\alpha}\left(P_{k}^{\text {succ }}\left(\boldsymbol{p}_{-r}\right)\right)
\end{aligned}
$$

subject to $\left(\boldsymbol{p}_{r}, \boldsymbol{p}_{-r}\right) \in \mathcal{P}$, 


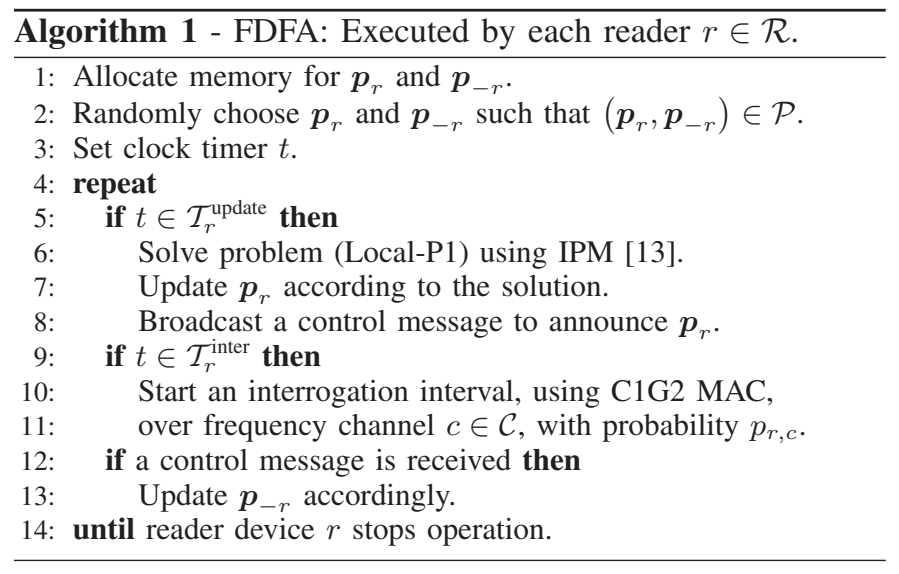

where $\succeq$ denotes coordinate-wise inequality. Note that the objective functions in problems (Local-P1) and (P1) are the same. For the objective function in (Local-P1), the first term is increasing in $\boldsymbol{p}_{r}$. The second and third terms are decreasing in $\boldsymbol{p}_{r}$. The last term does not depend on $\boldsymbol{p}_{r}$ as for each reader $k$, such that $r \notin \mathcal{S}_{k} \cup \mathcal{I}_{k}$, probability $P_{k}^{\text {succ }}$ does not depend on $\boldsymbol{p}_{r}$. By solving problem (Local-P1), reader $r$ can select $\boldsymbol{p}_{r}$ such that the objective function in problem (P1) is maximized assuming that $\boldsymbol{p}_{-r}$ is fixed (i.e., none of the other readers change their interrogation probabilities).

Theorem 2: For each reader $r \in \mathcal{R}$, problem (Local-P1) is a convex optimization problem.

The proof of Theorem 2 is given in Appendix B. From Theorem 2 , we can use various convex programming techniques to solve problem (Local-P1) in each reader $r \in \mathcal{R}$. In particular, problem (Local-P1) can be solved using the interior-point method (IPM) [13, Ch. 11] via local iterations if each reader can obtain the information on the interrogation probabilities of all readers within $d_{\max }^{I}=\max _{n \in \mathcal{R}} d_{n}^{I}$ distance away. Thus, the iterative coordinate ascent method is applicable.

\section{B. FDFA Algorithm}

Our proposed fully distributed frequency allocation (FDFA) algorithm for RFID systems is given in Algorithm 1. It is executed in each reader $r \in \mathcal{R}$. Let $\mathcal{T}_{r}^{\text {inter }}$ be an unbounded set of time instances at which reader $r$ may start an interrogation interval based on its interrogation probability vector $\boldsymbol{p}_{r}$. For any two consecutive elements $t_{1}, t_{2} \in \mathcal{T}_{r}^{\text {inter }}$, we have $\mid t_{2}-$ $t_{1} \mid=T$. Let $\mathcal{T}_{r}^{\text {update }}$ be an unbounded set of time instances at which reader $r$ updates its interrogation probability vector $\boldsymbol{p}_{r}$ by solving problem (Local-P1) using IPM. We assume that: (a) The updates are asynchronous across the readers. That is, for any $r, n \in \mathcal{R}$ such that $r \neq n$, we have: $\mathcal{T}_{r}^{\text {update }} \cap \mathcal{T}_{n}^{\text {update }}=\{\}$. (b) There is a global constant $T^{\text {update }}$ such that for any $r \in \mathcal{R}$, there exist $t_{1}, t_{2} \in \mathcal{T}_{r}^{\text {update }}$ such that $\left|t_{1}-t_{2}\right| \leq T^{\text {update }}$. In other words, all readers update their interrogation probabilities at least once every $T^{\text {update }}$ time units.

\section{Convergence and Optimality}

In this section, we analytically investigate the convergence and optimality features of Algorithm 1. At each time instance $t \geq 0$, let $F_{\alpha}(t)$ denote the current value of the objective function for problem (P1). We can show the following:
Theorem 3: For any choice of system parameters and starting from any initial point:

(a) Function $F_{\alpha}(t)$ is upper bounded; i.e., $F_{\alpha}(t) \leq-\frac{R}{\alpha} \leq 0$. (b) Function $F_{\alpha}(t)$ is non-decreasing at time $t \geq T^{\text {update }}$. That is, for each $t_{1}, t_{2} \geq T^{\text {update }}$ such that $t_{1} \leq t_{2}, F_{\alpha}\left(t_{1}\right) \leq F_{\alpha}\left(t_{2}\right)$. (c) Algorithm 1 converges. That is, there exists a $F_{\alpha}^{*}$ such that $F_{\alpha}^{*}=\lim _{t \rightarrow \infty} F_{\alpha}(t)$.

The proof of Theorem 3 is given in Appendix C. Theorem 3 guarantees the convergence of Algorithm 1 for any choice of system parameters. We can further show that:

Theorem 4: Any fixed point of Algorithm 1 is a stationary point of problem (P1). That is, it is at least a locally optimal solution for the non-convex optimization problem (P1).

The proof of Theorem 4 is given in Appendix D. From Theorems 3 and 4, convergence and local optimality of Algorithm 1 are guaranteed. Clearly, the obtained interrogation probabilities may not necessarily be globally optimal. However, simulation results in Section IV show that Algorithm 1 usually results in near globally optimal performance, making it a practical distributed frequency selection and randomized interrogation algorithm for large-scale RFID systems.

\section{Performance Evaluation}

In this section, we evaluate the performance of the proposed FDFA algorithm and compare it with four other distributed randomized interrogation schemes. In the simulation model, the total coverage area is a large square warehouse with each side equal to $1 \mathrm{~km}$. There are 25 readers randomly deployed in the field such that complete interrogation coverage is achieved. Clearly, some spots can be within the interrogation areas of multiple readers. For each reader $r$, the interrogation range $d_{r}^{R}$ and the interference range $d_{r}^{I}$ are $50 \mathrm{~m}$ and $100 \mathrm{~m}$, respectively [14]. On average, there are 1,000 mobile tags in the interrogation range of each reader. The interrogation interval $T$ is $10 \mathrm{sec}$. We also set $\alpha=10$. The average time for a complete successful interrogation process is $3 \mathrm{sec}$, assuming that the enhanced dynamic framed slotted Aloha [10] is being used. We also set $\tau^{\text {slot }}$ equal to $1 \mathrm{~ms}$ as in [4]. Problem (Local$\mathrm{P} 1$ ) is solved by using the MOSEK optimization software [15].

We start by first comparing the FDFA algorithm with DIA [7], S-LBT [8], and random and naive algorithms [6] in terms of the average probability of a successful interrogation among all readers (i.e., the ratio of the successfully interrogated tags compared to the total number of tags in the system). The simulation time is $1,000 \mathrm{sec}$. The results when the number of channels $C$ varies from 1 to 15 are shown in Fig. 5. In this figure, each point represents the average results of simulating 20 different randomly generated topologies. We can see that, for all algorithms, the probabilities increase as more channels become available. However, the FDFA algorithm always outperforms the other heuristic algorithms. Notice that all algorithms reach some saturation levels, which are different for all algorithms. Recall from Section II that reader-to-reader and type 1 reader-to-tag collisions can be avoided if the neighboring readers operate on different channels. However, having the readers operate on different channels cannot avoid type 2 reader-to-tag collision. Thus, if the number of channels 


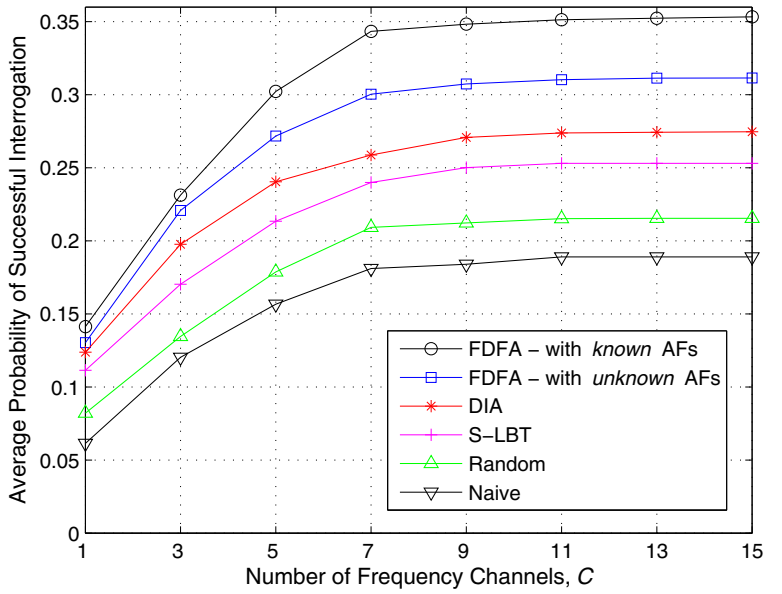

Fig. 5. Comparison between the proposed FDFA algorithm and DIA [7], S-LBT [8], and also random, and naive [6] algorithms when the number of channels varies from 1 to 15 . FDFA outperforms all other heuristic algorithms.

is high, different algorithms differ depending on their type 2 reader-to-tag collision avoidance. From Fig. 5, FDFA better avoids type 2 reader-to-tag collisions than the other algorithms.

Next, we investigate fairness (i.e., balanced performance) among readers. Following the same simulation model as in [7], [8], we assume that the number of channels $C$ is 10 . The simulation time is $1,000 \mathrm{sec}$. We compare the minimum number of successful interrogations among readers for different algorithms. Results are shown in Fig. 6. Each curve represents the average results of simulating 20 different randomly generated topologies. We can see that the FDFA algorithm performs better than all the heuristic algorithms as max-min fairness is indeed the design objective for FDFA. Using FDFA, if the asynchronism factors (AFs) (i.e., $\Delta_{r, n}$ for all $r, n \in \mathcal{R}$ ) are unknown, the minimum number of successful interrogations among all readers, which is measured at the end of the simulation, i.e., after 1000 time slots, is 23 . We notice that since each interrogation interval $T$ takes $10 \mathrm{sec}$, there are in total $1000 / 10=100$ interrogation attempts during the simulation time. Since, in the worst case, 23 out of 100 attempts are successful, the minimum probability of successful interrogation among all readers is 0.23 . This is $29 \%, 47 \%, 63 \%$, and $121 \%$ higher than a similar value for DIA, S-LBT, random, and naive algorithms, respectively. If the AFs are known, then the minimum probability of successful interrogation further increases by $11 \%$. Notice that by achieving max-min fairness, the processing load becomes similar for all reader devices.

Finally, we investigate optimality. Recall that the FDFA algorithm aims to maximize the objective value of the max-min fairness problem (i.e., $\sum_{r \in \mathcal{R}} f_{\alpha}\left(P_{r}^{\text {succ }}(\boldsymbol{p})\right)$ ). We determine the percentage difference of the optimal values obtained from the FDFA algorithm to the global optimal value of problem (P1). We consider 20 random topologies, each has 25 readers and 10 channels. The global optimum of problem (P1) is approximately obtained by running the FDFA algorithm 100 times, with each time starting from a different randomly selected initial point. The global optimal value is then selected to be the maximum observed local optimum among all 100 simulations. Results are shown in Fig. 7. On average, FDFA achieves $95.1 \%$

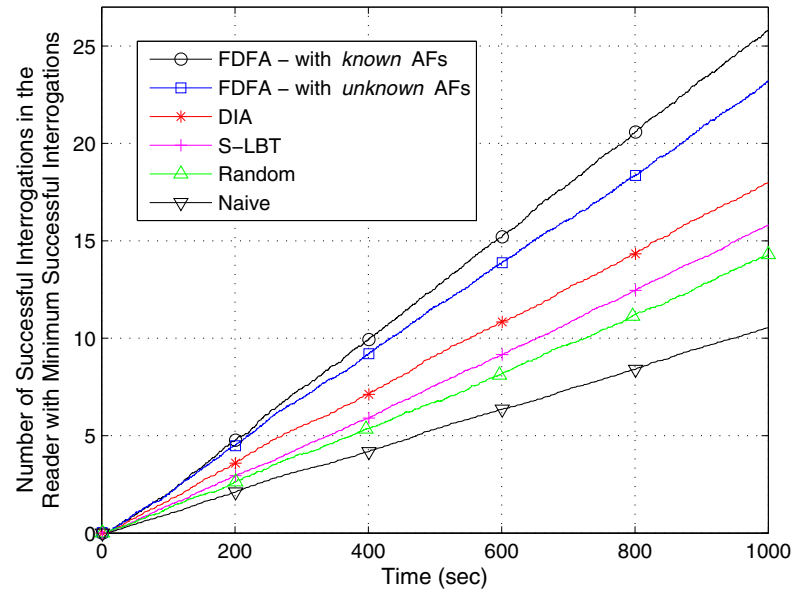

Fig. 6. Comparison between FDFA algorithm with the DIA [7], S-LBT [8], and also random, and naive [6] algorithms in terms of max-min fairness.

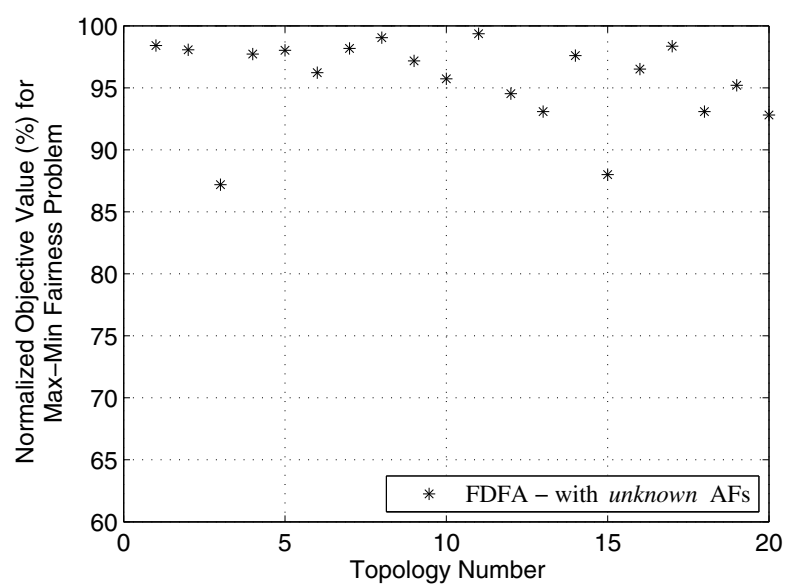

Fig. 7. Optimality of FDFA algorithm in terms of maximizing the objective value for max-min fairness problem (P1).

of the global optimal value of problem (P1). This implies near optimal performance for the FDFA algorithm.

\section{CONCLUSION}

In this paper, we systematically studied the randomized multi-channel interrogation problem for large-scale and dense RFID systems. We first modeled the reader-to-reader collision and reader-to-tag collisions (both types) in RFID systems. We then derived the probability of performing a successful interrogation by each reader, where the readers operate asynchronously. The joint channel selection and randomized interrogation problem was formulated as a max-min fair resource allocation problem. We proposed a distributed algorithm, called FDFA, to solve the optimization problem. FDFA is guaranteed to converge to at least a local optimal solution of the max-min fairness problem. Simulation results show that FDFA has a significantly better performance compared to the previously proposed heuristic multi-channel anti-collision algorithms in terms of the number of correct interrogations and fairness among readers. It also better utilizes the frequency spectrum and has a fast convergence speed. 


\section{APPENDIX}

\section{A. Proof of Theorem 1}

We first prove (9). Given (7), for each pair $r, n \in \mathcal{R}$, an interrogation cycle (which consists of all interrogation frames within an interrogation interval) for reader $n$ (with duration $\tau_{n}\left(N_{n}\right)$ ) may overlap with only one interrogation cycle of reader $r$ (with duration $\tau_{r}\left(N_{r}\right)$ ). From Fig. 4, overlapping of interrogation cycles between readers $r$ and $n$ occurs when

$$
-\tau_{n}\left(N_{n}\right)<\Delta_{r, n}<\tau_{r}\left(N_{r}\right) .
$$

Since readers randomly and independently start up their operation, $\Delta_{r, n}$ has a uniform distribution over $-T / 2$ and $T / 2$. Thus, the probability of (12) happening is as in (9). Next, we notice that for each reader $r \in \mathcal{R}$, the probability of completing a successful interrogation is obtained as

$$
P_{r}^{\mathrm{succ}}(\boldsymbol{p})=\sum_{c \in \mathcal{C}} p_{r, c} \mathbb{P}\left(A_{r, c}^{\mathrm{NoRR}} \cap A_{r, c}^{\mathrm{NoRT} 1} \cap A_{r, c}^{\mathrm{NoRT} 2}\right),
$$

where $\mathbb{P}\left(A_{r, c}^{\mathrm{NoRR}} \cap A_{r, c}^{\mathrm{NoRT} 1} \cap A_{r, c}^{\mathrm{NoRT} 2}\right)$ denotes the probability that no reader collision occurs while reader $r$ is performing an interrogation on channel $c . A_{r, c}^{\mathrm{NoRR}}, A_{r, c}^{\mathrm{NoRT} 1}$, and $A_{r, c}^{\mathrm{NoRT} 2}$ correspond to the events where no reader-to-reader collisions, no type 1 reader-to-tag collisions, and no type 2 reader-to-tag collisions occur, respectively. Since $\mathcal{V}_{r} \subseteq \mathcal{I}_{r} \cup \mathcal{S}_{r}$, if readerto-tag collisions do not happen, then reader-to-reader collision cannot happen either. Thus, we have

$$
\mathbb{P}\left(A_{r, c}^{\mathrm{NoRR}} \cap A_{r, c}^{\mathrm{NoRT} 1} \cap A_{r, c}^{\mathrm{NoRT} 2}\right)=\mathbb{P}\left(A_{r, c}^{\mathrm{NoRT} 1} \cap A_{r, c}^{\mathrm{NoRT} 2}\right) .
$$

Since $A_{r, c}^{\mathrm{NoRT} 1}$ and $A_{r, c}^{\mathrm{NoRT} 2}$ are independent events due to (3), we have: $\mathbb{P}\left(A_{r, c}^{\mathrm{NoRT} 1} \cap A_{r, c}^{\mathrm{NoRT} 2}\right)=\mathbb{P}\left(A_{r, c}^{\mathrm{NoRT} 1}\right) \mathbb{P}\left(A_{r, c}^{\mathrm{NoRT} 2}\right)$. Finally, for all $c \in \mathcal{C}$, we have $\mathbb{P}\left(A_{r, c}^{\mathrm{NoRT} 1}\right)=\prod_{n \in \mathcal{I}_{r}}\left(1-\gamma_{r, n} p_{n, c}\right)$ and $\mathbb{P}\left(A_{r, c}^{\mathrm{NoRT} 2}\right)=\prod_{n \in \mathcal{S}_{r}}\left(1-\gamma_{r, n} \sum_{e \in \mathcal{C}} p_{n, e}\right)$. Replacing these probabilities in (13), we obtain (8).

\section{B. Proof of Theorem 2}

From (8), the objective function in (Local-P1) becomes

$$
\begin{aligned}
& f_{\alpha}\left(\sum_{c \in \mathcal{C}} \theta_{r, c} p_{r, c}\right)+\sum_{n: r \in \mathcal{S}_{n}} f_{\alpha}\left(\vartheta_{r, n}\left(1-\gamma_{r, n} \sum_{c \in \mathcal{C}} p_{r, c}\right)\right) \\
& +\sum_{m: r \in \mathcal{I}_{m}} f_{\alpha}\left(\sum_{c \in \mathcal{C}} \zeta_{r, m, c}\left(1-\gamma_{r, m} p_{r, c}\right)\right)+\xi_{r},
\end{aligned}
$$

where $\theta_{r, c}, \vartheta_{r, n}, \zeta_{r, m, c}$, and $\xi_{r}$ only depend on $\boldsymbol{p}_{-r}$ and can be treated as constants in problem (Local-P1). For example, $\theta_{r, c}=\left(\prod_{i \in \mathcal{S}_{r}}\left(1-\gamma_{r, i} \sum_{e \in \mathcal{C}} p_{i, e}\right)\right)\left(\prod_{j \in \mathcal{I}_{r}}\left(1-\gamma_{r, j} p_{j, c}\right)\right)$. Since $f_{\alpha}$ is concave for $r \in \mathcal{R}$, the objective function of problem (Local-P1) is a summation of concave-affine compositions over $\boldsymbol{p}_{r}$. Thus, it is concave. Since the constraint in (Local-P1) is linear, problem (Local-P1) is a convex problem.

\section{Proof of Theorem 3}

Part (a): Since $P_{r}^{\text {succ }} \leq 1, f_{\alpha}\left(P_{r}^{\text {succ }}\right) \leq f_{\alpha}(1)=-1 / \alpha$.

Part (b): We prove this part by contradiction. First, we assume that $F_{\alpha}\left(t_{1}\right)>F_{\alpha}\left(t_{2}\right)$. In that case, there exists a time instance $t \in\left[t_{1}, t_{2}\right]$ such that running Algorithm 1 results in reducing the value of the objective function of problem (P1) at time $t$. In other words, there exists a reader $r \in \mathcal{R}$ such that $t \in \mathcal{T}_{r}^{\text {update }}$ and $F_{\alpha}$ is reduced by executing line 6 of Algorithm 1 in reader $r$. However, this is impossible as the objective function in problem (P1) is the same as that in problem (Local-P1). Thus, we indeed have $F_{\alpha}\left(t_{1}\right) \leq F_{\alpha}\left(t_{2}\right)$.
Part (c): The limit in this part directly results from Parts (a) and (b). Notice that any upper bounded non-decreasing sequence of real numbers converges to a fixed point.

\section{Proof of Theorem 4}

Let $\boldsymbol{p}^{*}$ denote any fixed point of Algorithm 1. Given $\boldsymbol{p}_{-r}=\boldsymbol{p}_{-r}^{*}$ for $r \in \mathcal{R}, \boldsymbol{p}_{r}=\boldsymbol{p}_{r}^{*}$ is the optimal solution of problem (Local-P1). Since (Local-P1) is convex, $\boldsymbol{p}^{*}$ should satisfy the Karush-Kuhn-Tucker (KKT) conditions [13, p. 244] corresponding to (Local-P1) for all $r \in \mathcal{R}$. By definition, each stationary point [16, p. 194] of non-convex problem (P1) should also satisfy all the KKT conditions for problem (P1). Since the objective functions in problems (P1) and (Local$\mathrm{P} 1)$ are the same and the set of constraints in (P1) is the union of the set of constraints in (Local-P1) for all $r \in \mathcal{R}$, the KKT conditions for non-convex problem (P1) are equal to the union of the KKT conditions for problem (Local-P1) for all $r \in \mathcal{R}$. Thus, since $\boldsymbol{p}^{*}$ satisfies the KKT conditions of problem (Local-P1) for all readers, it also satisfies the KKT conditions for problem (P1). That is, each fixed point $\boldsymbol{p}^{*}$ is a local optimal solution for problem (P1).

\section{ACKNOWLEDGEMENT}

This research is supported by the Natural Sciences and Engineering Research Council (NSERC) of Canada under grant number STPGP 364962-08.

\section{REFERENCES}

[1] V. Shah-Mansouri and V. W. S. Wong, "Anonymous cardinality estimation in RFID systems with multiple readers," in Proc. of IEEE Globecom, Honolulu, HI, Dec. 2009

[2] J. S. Choi, H. Lee, D. W. Engels, and R. Elmasri, "Robust and dynamic bin slotted anti-collision algorithms in RFID systems," in Proc. of IEEE Int'l Conf. on RFID, Las Vegas, NV, Apr. 2008.

[3] H. Koh, S. Yun, and H. Kim, "Sidewalk: a RFID tag anti-collision algorithm exploiting sequential arrangements of tags," in Proc. of IEEE ICC, Beijing, China, May 2008.

[4] EPCglobal, "Class 1 Generation 2 UHF air interface protocol standard, v. 1.1.0," Dec. 2005. [Online]. Available: http://www.epcglobalinc.org/standards/uhfc1g2

[5] Z. Zhou, H. Gupta, S. R. Das, and X. Zhu, "Slotted scheduled tag access in multi-reader RFID systems," in Proc. of IEEE Int'l Conf. on Networks Protocols (ICNP), Beijing, China, Sept. 2007.

[6] S. Jain and S. R. Das, "Collision avoidance in a dense RFID network," in Proc. of ACM Int'l Workshop on Wireless Network Testbeds, Experimental Evaluation and Characterization, Los Angeles, CA, Sept. 2006.

[7] Y. Tanaka and I. Sasase, "Interference avoidance algorithms for passive RFID systems using contention-based transmit abortion," IEICE Trans. on Communications, vol. E90-B, pp. 3170-3180, Nov. 2007.

[8] C.-H. Quan, J.-C. Choi, G.-Y. Choi, and C.-W. Lee, "The Slotted-LBT: A RFID reader medium access scheme in dense reader environments," in Proc. of IEEE Int'l Conf. on RFID, Las Vegas, NV, Apr. 2008.

[9] EPCglobal, "Low level reader protocol," Aug. 2007. [Online]. Available: http://www.epcglobalinc.org/standards/llrp

[10] S.-R. Lee, S.-D. Joo, and C.-W. Lee, "An enhanced dynamic framed slotted ALOHA algorithm for RFID tag identification," in Proc. of Int'l Conf. on Mobile and Ubiquitous Systems, San Diego, CA, July 2005.

[11] J. Mo and J. Warland, "Fair end-to-end window-based congestion control," IEEE/ACM Trans. Networking, vol. 8, pp. 556-567, Oct. 2000.

[12] D. P. Bertsekas and J. N. Tsitsiklis, Parallel and Distributed Computation: Numerical Methods. Prentice Hall, 1989.

[13] S. Boyd and L. Vandenberghe, Convex Optimization. Cambridge University Press, 2004.

[14] S. Ahson and M. Ilyas, RFID Handbook: Applications, Technology, Security, and Privacy. CRC Press, 2008.

[15] "MOSEK." http://www.mosek.com, 2009.

[16] D. P. Bertsekas, Nonlinear Programming, 2nd ed. Athena Sci., 2004. 